\title{
Association between individual surgeon volume and outcome in mitral valve surgery: a systematic review
}

\author{
Berdel Akmaz ${ }^{1,2}$, Sander M. J. van Kuijk ${ }^{3}$, Peyman Sardari Nia ${ }^{2}$ \\ ${ }^{1}$ Faculty of Health Medicine and Life Sciences, Maastricht University, Maastricht, The Netherlands; ${ }^{2}$ Department of Cardiothoracic Surgery, \\ Maastricht University Medical Centre, Maastricht, The Netherlands; ${ }^{3}$ Department of Clinical Epidemiology and Medical Technology Assessment \\ (KEMTA), Maastricht University Medical Center (MUMC+), Maastricht, The Netherlands \\ Contributions: (I) Conception and design: B Akmaz, P Sardari Nia; (II) Administrative support: B Akmaz, P Sardari Nia; (III) Provision of study \\ materials or patients: None; (IV) Collection and assembly of data: B Akmaz; (V) Data analysis and interpretation: All authors; (VI) Manuscript \\ writing: All authors; (VII) Final approval of manuscript: All authors. \\ Correspondence to: Peyman Sardari Nia, MD, PhD. Department of Cardiothoracic Surgery, Maastricht University Medical Center, P. Debyelaan 25, \\ Maastricht, 6202 AZ, The Netherlands. Email: peyman.sardarinia@mumc.nl.
}

\begin{abstract}
Background: Surgeon volume has been identified as a possible factor that influences outcomes in mitral valve (MV) surgery. The aim of this study was to systematically review all published studies on the association between individual surgeon volume and outcome in MV surgery.

Methods: PubMed was searched last on 19 November 2020. The reporting of this systematic review was done in accordance with PRISMA guidelines. Manuscripts were eligible when these studied individual surgeon volumes and its association with repair rate, mortality or reoperation. The methodological quality of the studies was assessed with the Newcastle-Ottawa Scale (NOS). Absolute numbers and percentages of the outcome measures, odds ratios (ORs), $\mathrm{P}$ values and threshold values regarding surgeon volume were collected.
\end{abstract}

Results: A total of 7 retrospective cohort studies were included in the qualitative analysis with total of 158488 patients. Definitions of surgeon volumes were found to be heterogenic and therefore pooling of data was not possible. Surgeon volume was significantly associated with repair rate $(\mathrm{OR}=1.25-5.5)$ and mortality ( $\mathrm{OR}=0.46-0.84$ and $\mathrm{OR}=1.50-2.27$ depending on the reference group). Regarding reoperation, results were not consistent and did not always show a significant lower reoperation rate when surgeon volume increased. A mean threshold of minimally $30 \mathrm{MV}$ surgeries per year was found.

Discussion: Higher surgeon volume is significantly associated with improved outcomes of repair rate and mortality. MV should preferentially be performed by high-volume surgeons and centralization of MV surgery might be necessary.

Keywords: Mitral valve surgery (MV surgery); surgeon volume; mortality; reoperation; repair rate.

Submitted Apr 01, 2021. Accepted for publication Jun 04, 2021.

doi: $10.21037 /$ jtd-21-578

View this article at: https://dx.doi.org/10.21037/jtd-21-578

\section{Introduction}

The burden of valvular heart diseases in the general population is considerable and its prevalence is estimated at $2.5 \%$ (1). Mitral valve (MV) repair is considered the gold standard for treating degenerative $\mathrm{MV}$ disease and is superior to MV replacement (2-6). MV repair is performed using full median sternotomy or minithoracotomy (with endoscopic or robotic assistance). The durability of the repair and the repair rate are dependent on the surgeon's experience and the nature of the MV pathology (2).

Literature shows an association between hospital volume and outcomes in MV surgery $(7,8)$. Highervolume hospitals were found to have lower operative mortality rates than smaller hospitals for MV surgery. 
High individual surgeon's volume might also increase the likelihood of durable MV repair, the repair rate and improved outcomes $(7,9)$. Surgeon experience accounts for a large amount of the effect hospital volume has on outcomes and is associated with mortality in cardiovascular procedures (10). Surgeon volumes may therefore be more important than hospital volumes $(8,9,11)$. It was proposed that surgeons need a volume of $25 \mathrm{MV}$ repairs per year to provide consistency in their outcome. However, these numbers are based on thresholds of urological malignancy in the UK which might not be representative for valve surgery $(8,12)$.

Despite the possible association between surgeon volume and outcomes, no consensus on the association between surgeon volume and outcomes for MV surgery exists $(8,12)$. In the literature, several systematic reviews regarding surgeon volume for other surgical specialties have been performed $(13,14)$. However, reviews considering the relationship between surgeon volume and outcomes in MV surgery are still lacking $(11,15)$. Therefore, the aim of this study is to systematically review all published studies on the association between individual surgeon volume and outcome in adult MV surgery patients.

We present the following article in accordance with the Preferred Reporting Items for Systematic Reviews and Meta-Analysis (PRISMA) reporting checklist (16) (available at https://dx.doi.org/10.21037/jtd-21-578).

\section{Methods}

A study protocol was designed and used based on the guidelines described in the Cochrane Reviewers' Handbook (17). Inclusion and exclusion criteria, outcome measures and methods of the analysis were described in the protocol.

\section{Participants, intervention and outcomes}

Manuscripts were included when these studied individual surgeon volume and its association with one of the predefined outcome measures. Surgeon volumes were defined as low-volume or high-volume using the volume categories of each study and defining the lowest volume category in the studies as reference.

Outcome measures were repair rate, mortality and reoperation per surgeon volume. 'Repair rate' was defined as a percentage for the degree of repair of the MV, per surgeon volume group. 'Mortality' was defined per surgeon volume group as operative mortality, 30-day mortality or 1-year mortality, which differed per study. The outcome 'Reoperation' was defined in absolute numbers and percentages per surgeon volume group.

\section{Search strategy}

PubMed was searched for studies from 1978 up to November 2020 regarding the influence of individual surgeon volume on repair rate, mortality and reoperation in MV surgery patients. Additional studies were sought via Embase. The Cochrane review database was used to search for existing systematic reviews on this topic. Furthermore, reference lists of the articles were checked on relevant literature.

The search included the following $(\mathrm{MeSH})$ search terms: 'Mitral valve insufficiency', 'Mitral valve prolapse', 'Mitral valve stenosis', 'Mitral valve annuloplasty', 'Mitral valve surgery', 'Mitral valve repair', 'High-volume surgeons', 'Low-volume surgeons', 'Surgeon volume', 'Clinician volume', 'Physician volume', 'Cardiac Surgical Procedures/ mortality', 'Repair rate', 'Reoperation' and 'Recovery'. To reflect total annual mitral experience, the search included both MV repairs and replacements. The last search was run on 19 November 2020. The reproducible search string can be found in the Appendix 1.

\section{Study selection}

Articles were first screened for eligibility based on their titles and abstracts. Subsequently, full texts of the potentially eligible studies were read according to the predefined eligibility criteria. Articles reporting combined data were only included when data for MV surgery was presented separately. Experimental or cohort studies were included only. Studies were excluded when they were not available in English, not in full-text or when these considered hospital volumes instead of surgeon volumes. Furthermore, studies describing learning curves of (trainee) surgeons were excluded because these do not describe individual annual case volumes.

\section{Data extraction}

Data extraction tables were drafted by two authors independently, and information on the following study characteristics were collected for each article: study period, setting, study design, MV repair or replacement, 

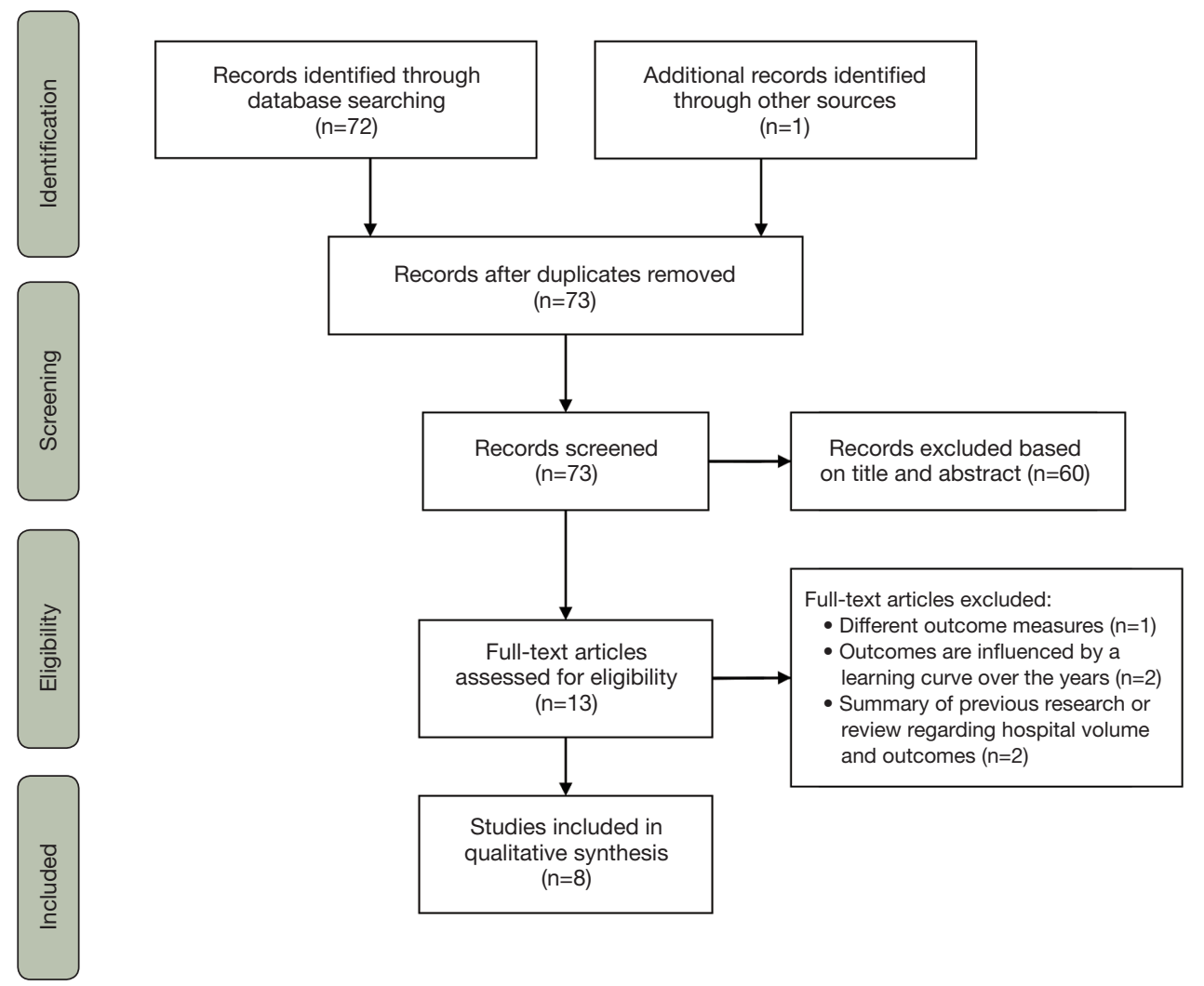

Figure 1 PRISMA flow diagram of the study selection. PRISMA, Preferred Reporting Items for Systematic Reviews and Meta-Analysis.

participants in total (male/female), age and outcome measure (repair rate, mortality, reoperation). Measures of association that were included are odds ratios (ORs), relative risks (RRs) and hazard ratios (HRs), including 95\% confidence intervals (CIs) and $\mathrm{P}$ values. In case these were not given, the appropriate measure of association was calculated. Furthermore, threshold values were included in case these were described. Assessment of study quality was done using the Newcastle-Ottawa Scale (NOS) quality assessment scale. Studies scoring 7 or higher were defined as high methodological quality. Studies scoring 5 or lower were considered as low methodological quality.

\section{Statistical analysis}

Definitions of surgeon volumes were found to be heterogeneous and therefore pooling of data was not possible. Data was described qualitatively reporting absolute numbers and ORs or HRs. If available, adjusted ORs (AORs) or HRs were reported. In case no numerical results were reported, numbers were estimated from published figures or scatter plots. Missing ORs were calculated for four studies: higher volume was defined as the 'exposed group', compared to a 'control group' with low volume.

\section{Results}

\section{Study selection}

Seventy-two articles were found on PubMed and used for this review. One additional study was found by relatedarticle search. No duplicates were included. After title and abstract screening, 13 full texts were assessed for eligibility (Figure 1). Five did not meet the eligibility criteria and were excluded because the measured outcomes were different than our predefined outcome measures; learning curves of surgeons were described instead of individual surgeon volumes or summaries were given of previously conducted research on hospital volume and outcomes (no systematic reviews). In total, eight reports were included but seven unique studies were identified, as two different reports described the same study $(18,19)$. 


\section{Study characteristics}

The characteristics of the included studies are described in Table 1. The included studies had been conducted in the United States and Australia. The procedures in included studies were obtained from cardiac surgery databases except for Chikwe et al. who used a database that collects data on every ambulatory surgery, hospital discharge and emergency department visit in New York State. The patients in the cardiac surgery databases used by the different studies overlap, since the US regions overlap. This probably accounts for a large proportion of the patients. Furthermore, the Society of Thoracic Surgeons Adult Cardiac Surgery Database has been used two times. The number of procedures per study ranged from 1,054 to 55,311. All studies were retrospective cohort studies and were conducted in the period between 2001-2016 (11,18-24). Repair rate was described in six studies $(18-21,23,24)$. Reoperation was only described by two studies $(20,21)$. Mortality was studied by five studies (11,20-23).

\section{Methodological study quality}

All studies scored the same on the 'selection' and 'comparability' category (Table 2). Only Ch'ng et al. did not score on comparability because they did not retrospectively compare two groups, comparing high- and low-volume surgeons such as in the other studies. Furthermore, six articles scored lower on the 'outcome' category due to lack of reported follow-up time (11,19,20,22-24). Moreover, it was unclear if all follow-up data was complete (19) or a description of lost follow-up data was lacking (23). Chikwe et al. scored highest on the NOS scale mainly because the authors applied a long follow-up time and thoroughly reported on missing data (21).

\section{The association between surgeon volume and repair rate}

Table 3 shows reported repair rates for different surgeon volume quartiles per study. Absolute numbers were missing in some studies and are therefore not reported. All ORs were adjusted for confounding variables that may have led to choosing to repair or not, except for Kilic et al. since its ORs were calculated by us (23). Bolling et al. and Kilic et al. both show in their articles that the effect of volume on repair rate was linear in the lower tail and flatter in the upper tail of the volume distribution, suggesting a plateau effect in the association $(19,23)$. Besides the comparison of attempted repair rates $(\mathrm{OR}=0.34)$, Badhwar et al. also shows an increased successful repair for higher volume surgeons $(\mathrm{OR}=0.62)(20)$.

Three ORs were not statistically significant (ORs $=1.17-1.27)(21-23)$, the remaining ORs were significant $(19-21,23,24)$.

\section{The association between mortality and surgeon volume}

Each study reported mortality for different surgeon volume quartiles (Table 4). The periods for the mortality rates also differed from operative to 4-year mortality. We studied mortality as perioperative mortality: operative mortality or 30-day mortality ( $\mathrm{n}=3$ ). Besides, we defined a group of 1 -year mortality and beyond $(n=3)(11,20-23,25)$. Survival estimates were translated into mortality rates (21).

All studies reported AORs, using risk-adjusted mortality and multivariable analyses. The given and calculated ORs, in most cases, were based on mortality rates of low-volume surgeons versus mortality rates of high-volume surgeons, using low-volume as the reference group. Two studies used high-volume as reference group in the calculation of the OR in their studies $(20,23)$. These differences in calculations should not be overlooked in the interpretation of Table 4 and led to ORs $<1$, rather than $>1$. The significant ORs $<1$ ranged from 0.15-0.84 and the ORs $>1$ ranged from 1.50-2.27.

Chikwe et al. showed that higher annual surgeon volume was significantly associated with lower mortality rates (OR =0.46), P-value was not given (21). Ch'ng et al. reported significantly $(\mathrm{OR}=0.84, \mathrm{P}=0.003$ ) less chance to die per 20 extra MV surgeries per surgeon annually. Decreasing linearity can be seen from $10 \mathrm{MVO}$ in their scatter plot. ORs per surgeon volume from the survival rates in the scatter plot were also significant $(\mathrm{ORs}=0.15-0.55)$ (11). Two studies also reported significantly 1.50-2.27 more chance to die perioperatively when operated by an annual low-volume surgeon $(20,23)$. All mortality rates decrease when surgeon volume increases, although one OR was not significant (22).

\section{The association between surgeon volume and reoperations}

The reoperation rates that were reported in two studies are shown in Table 5. Reoperation is a time dependent variable and was in both studies measured at 1 year. Chikwe et al. used an adjusted HR (AHR), Badhwar et al. did use HR. The difference in reoperation was not significant according to Badhwar et al., considering the CI (HR =1.14, CI: 0.60-2.18). The difference was small 


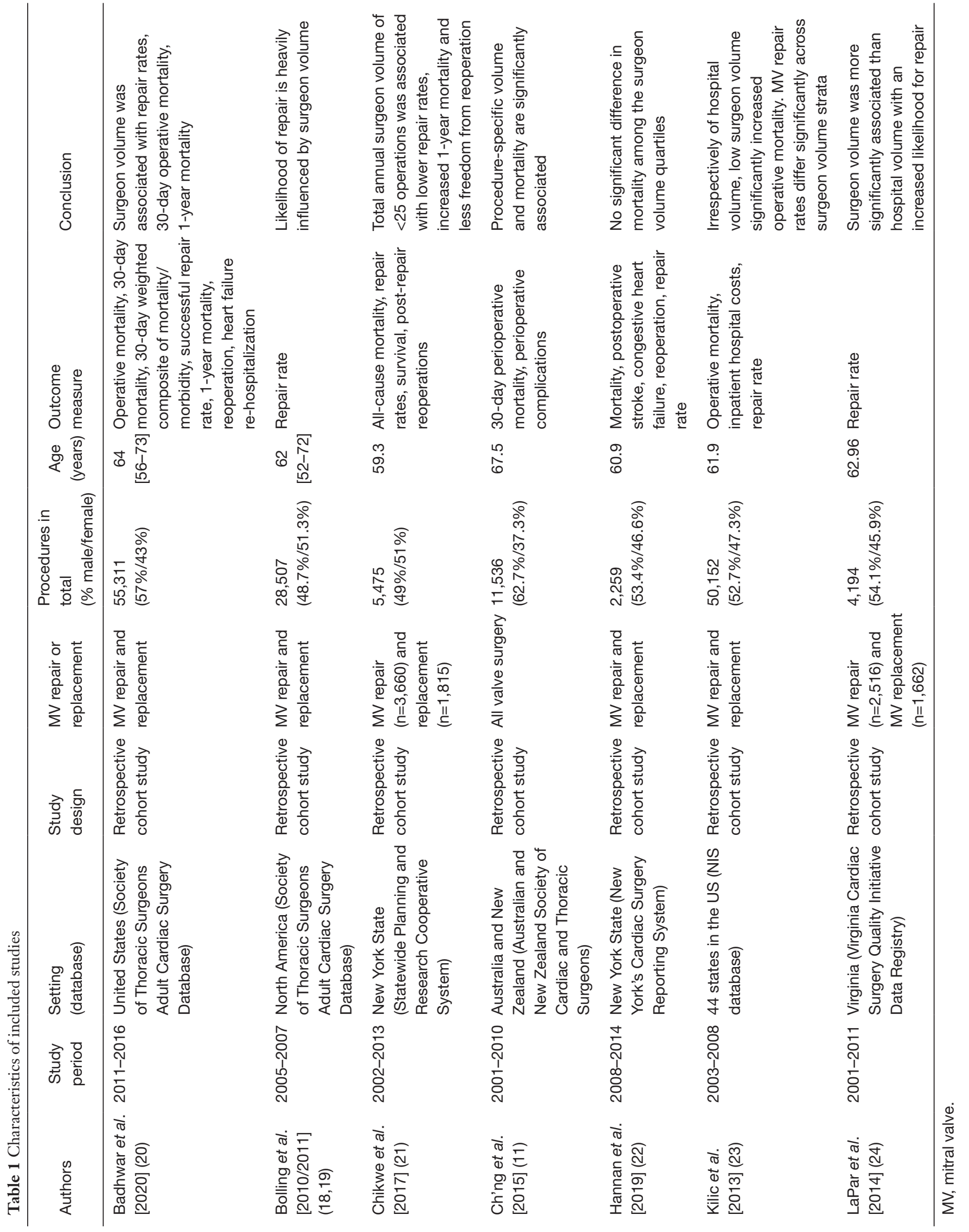


Table 2 Study quality assessment according to the NOS

\begin{tabular}{|c|c|c|c|c|c|}
\hline Authors & \multicolumn{5}{|c|}{ NOS } \\
\hline Badhwar et al. [2019] (20) & 4 & 1 & 2 & 7 & High \\
\hline Bolling et al. [2010] (19) & 4 & 1 & 1 & 6 & Fair \\
\hline Chikwe et al. [2017] (21) & 4 & 1 & 3 & 8 & High \\
\hline Hannan et al. [2019] (22) & 4 & 1 & 2 & 7 & High \\
\hline Kilic et al. [2013] (23) & 4 & 1 & 1 & 6 & Fair \\
\hline LaPar et al. [2014] (24) & 4 & 1 & 2 & 7 & High \\
\hline
\end{tabular}

and therefore clinically not relevant (20). Chikwe et al. reported a significant AHR of 0.45 comparing a surgeon volume of $>25$ with $<25$, meaning a strong decrease in risk of reoperation for patients that are operated by an annual high-volume surgeon (21). One report studied reoperation in MV repair and MV replacement but did not report these per surgeon volume. Therefore, this study was not included in Table 5 (22).

\section{Threshold values that might lead to better outcomes}

Four studies have proposed threshold values for minimum annual surgeon volumes that might lead to better $M V$ outcomes, see Table 6 . Bolling et al. only measured repair rate and proposed a minimal annual MV surgeon volume of 40 per year based on literature (19). Another study also defined an inflection point at 20 surgeries per year because surgeons performing $>20$ mitral operations per year were found to perform $M V$ repair three to four times more likely than surgeons performing $<20$ mitral operations per year (24). Another study which focused on all three outcomes, set a threshold at $25 \mathrm{MV}$ operations (MVO) per surgeon per year. Surgeons performing <25 MVO per year had significantly higher mortality rates, were significantly more likely to do MV replacements than $M V$ repairs and their patients were significantly more likely to undergo reoperation in long-term follow-up (21). Badhwar et al. defined an inflection point of 35 cases per year for an increase in attempted MV repair, successful MV repair and a decrease in 30-day mortality (20). All studies did find inflection points rather than linear relationships. The mean threshold of the four studies is $30 \mathrm{MV}$ surgeries per year.

\section{Discussion}

This is the first systematic literature review of the association between surgeon volume and outcomes such as repair rate, mortality and reoperation for $M V$ surgery. A total of 7 studies were included representing 158,488 patients in total. Principal findings were that surgeon volume is significantly associated with repair rate and mortality, but evidence regarding the outcome of reoperation is not sufficient since only two studies reported on this yet.

\section{Summary of evidence}

The ORs for the association between surgeon volume and repair rate ranged significantly from $1.25-5.5$, meaning that patients operated by a surgeon with a high surgeon volume, are 1.25-5.5 times as likely to undergo repair $(19-21,23,24)$. The plateau phase between increased surgeon volume and repair rate that was found in two studies, is also described in terms of a learning curve in the literature. For example, increasing total case volume for a surgeon in pancreaticoduodenectomies or radical prostatectomies does not lead to improvements in outcomes when a plateau in performance is reached. Reaching this plateau takes a certain amount of cases to successfully learn the procedure $(13,19,23)$.

ORs also showed that increased mitral surgeon volume was significantly associated with lower mortality rates, for instance meaning 54\% less chance to die for patients that are operated by a high-volume surgeon $(11,20,21,23)$. In one study the decrease in mortality for increasing surgeon volume was small and led to an OR that was not significant 
Table 3 Results of included studies regarding repair rate

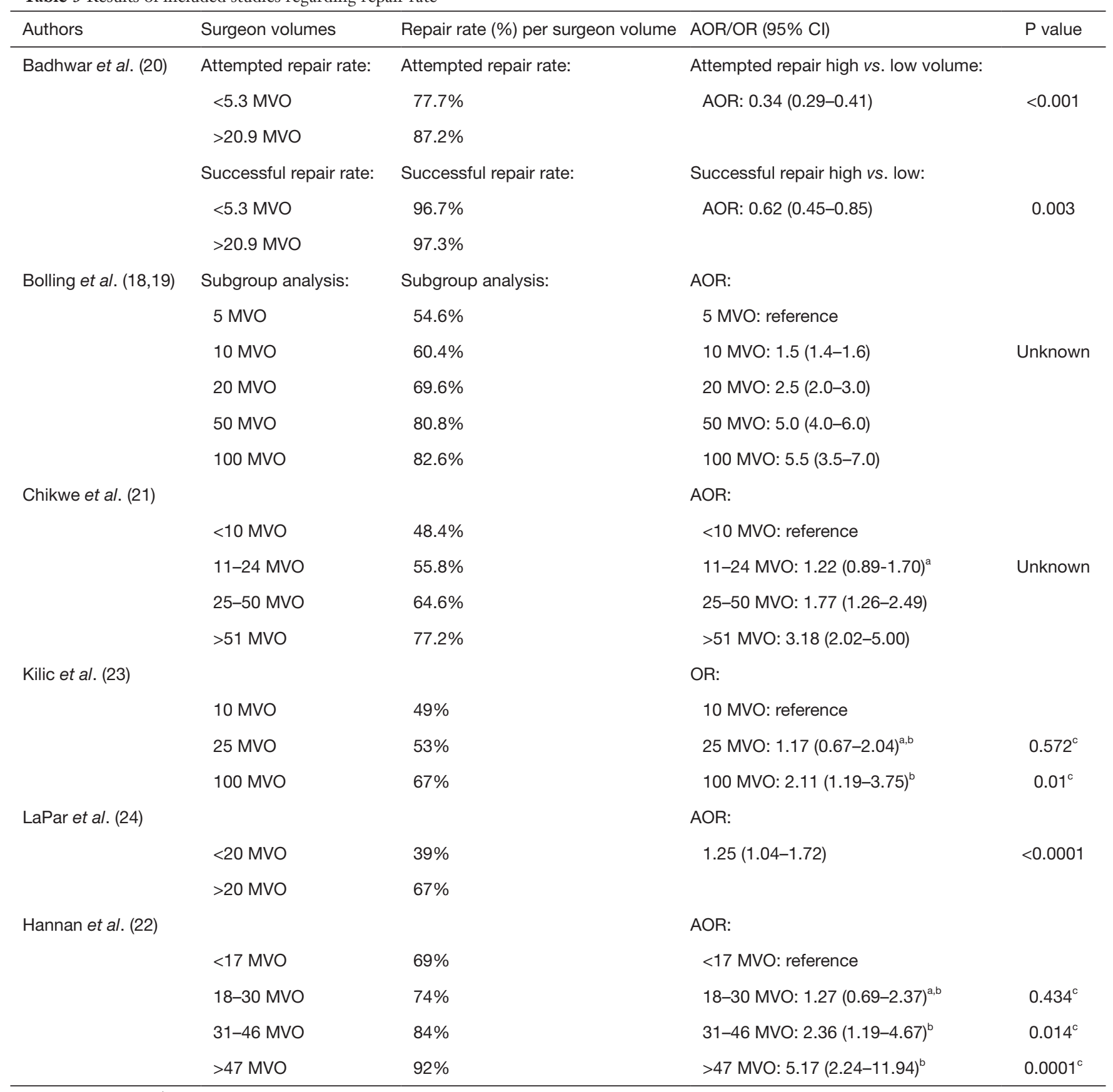

a, not significant; ${ }^{\mathrm{b}}$, OR was not given in the article and was calculated by the authors; ${ }^{\circ}, \mathrm{P}$ value was not given in the article and was calculated by the authors. AOR, adjusted odds ratio; $\mathrm{Cl}$, confidence interval; MVO, mitral valve operations; OR, odds ratio.

according to its $\mathrm{CI}$ and $\mathrm{P}$ value (22).

The significant association between surgeon volume and mortality accounts for other procedures in the literature as well. For example, the significant ORs for cardiovascular procedures such as aortic valve replacement and coronary- artery bypass grafting (CABG) ranged from 1.36-1.65 (10). Research regarding ruptured aortoiliac aneurysms for instance, abdominal aortic aneurysm repair and carotid endarterectomies also showed significantly lower mortality rates for high-volume surgeons (26-28). 
Table 4 Results of included studies regarding mortality

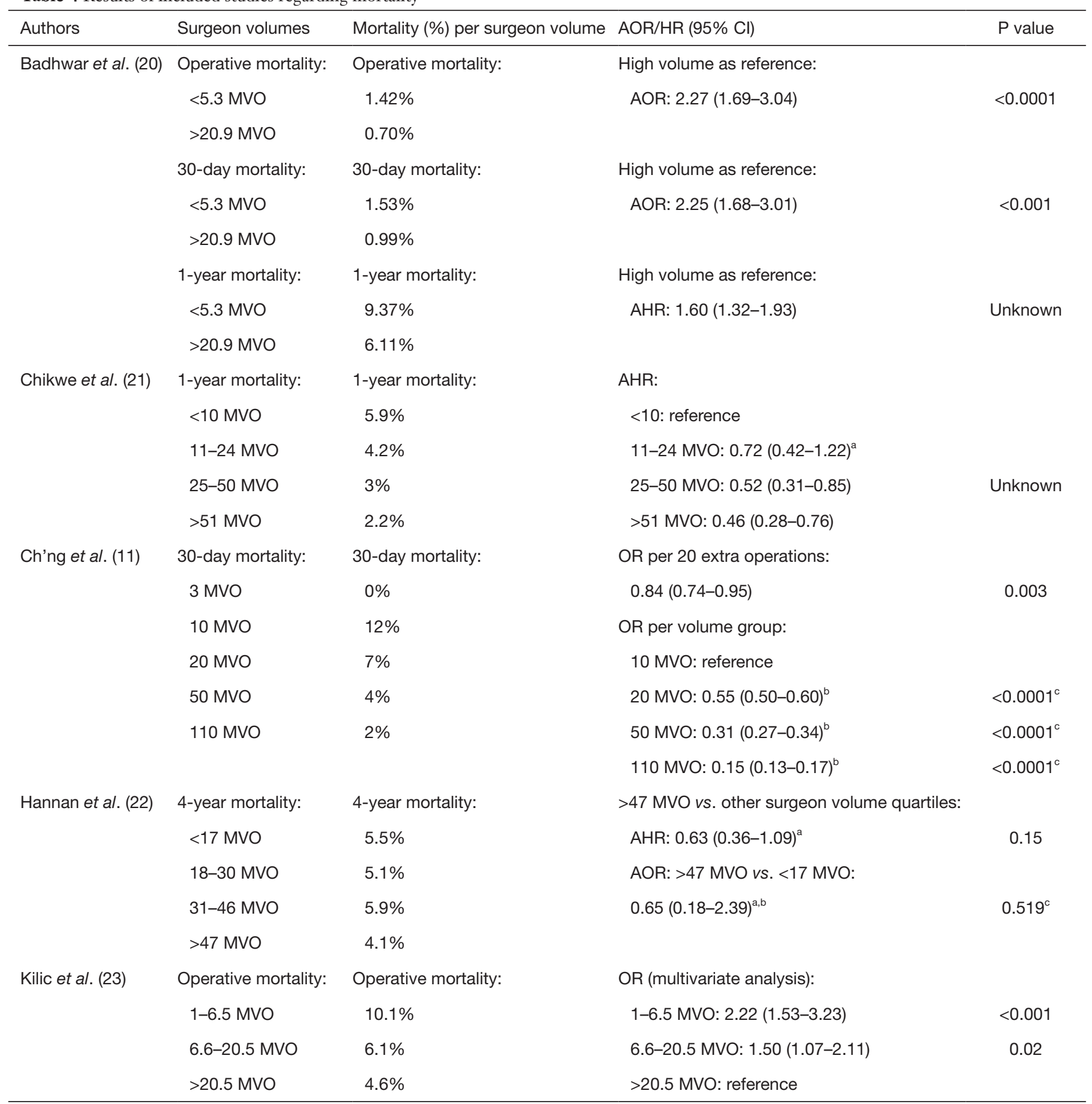

\footnotetext{
a , not significant; ${ }^{b}$, OR was not given in the article and was calculated by the authors; ${ }^{\mathrm{c}}$, $\mathrm{P}$ value was not given in the article and was calculated by the authors. AOR, adjusted odds ratio; HR, hazard ratio; Cl, confidence interval; MVO, mitral valve operations; OR, odds ratio.
} 
Table 5 Results of included studies regarding reoperations

\begin{tabular}{lccc}
\hline Authors & Surgeon volumes & Freedom from reoperation (\%) at 1 year & AHR/HR (95\% Cl) \\
\hline Badhwar et al. (20) & $<5.3 \mathrm{MVO}$ & 1.07 & HR: $1.14(0.60-2.18)^{\mathrm{a}}$ \\
& $>20.9 \mathrm{MVO}$ & 0.95 & \\
& $<25 \mathrm{MVO}$ & 3.6 & AHR: $0.45(0.26-0.76)$ \\
Chikwe et al. (21) & $>25 \mathrm{MVO}$ & 1.3 & 0.003 \\
\end{tabular}

${ }^{\mathrm{a}}$, not significant. AHR, adjusted hazard ratio; HR, hazard ratio; $\mathrm{Cl}$, confidence interval; MVO, mitral valve operations.

Table 6 Proposed threshold values that led to better outcomes

\begin{tabular}{llc}
\hline Study & Outcome measures & Threshold value \\
\hline Bolling et al. & Repair rate & 40 \\
LaPar et al. & Repair rate & 20 \\
Chikwe et al. & Repair rate, mortality, & 25 \\
& reoperation & \\
Badhwar et al. & Repair rate, mortality & 35 \\
\hline
\end{tabular}

Regarding reoperation, one HR was not significant, and another study reported a strong decrease in risk for reoperation for patients that are operated by an annual high-volume surgeon. No conclusions can be drawn regarding reoperation based on these studies $(20,21)$. Another study showed that low surgeon volume was significantly associated with a higher reoperation rate for lumbar spine surgeries (29). The same result was found for inflatable penile prosthesis surgeries and open incisional hernia repairs $(30,31)$. Whether outcomes are influenced by surgeon volume or hospital volume, depends largely on the sort of procedure. This is influenced by the degree of technical skill and the use of specific intraoperative processes for a particular surgery (10).

Since surgeon volume influences outcomes significantly, it is important to define a threshold annual surgeon volume that might lead to better patient outcomes. The mean threshold that can be derived from the four thresholds in this systematic review is $30 \mathrm{MV}$ surgeries per year. This corresponds to the threshold that was defined by Bridgewater $e t$ al., who developed standards for best practice by an analysis of existing literature and discussion with multidisciplinary panel. They concluded that surgeons should do at least $25 \mathrm{MV}$ repairs per year (8). Annual surgeon volumes were also recommended for other surgeries in the literature, such as 13-21 per year for colorectal cancer, 11 for angioplasty and 75 for pediatric cardiac surgery to achieve significantly better outcomes (14).

Although this review concludes for a threshold of a MV surgeon volume of 30 per year, it remains problematic to define an arbitrary cut-off. For example, a surgeon with 25 repair per year for the past two years, may be just as qualified as a new graduate with an experience of 100 mitral repairs in the past 2 years (12). It is recommended to investigate the difference between low-volume and high-volume groups using arbitrary cut-offs in future research.

\section{Limitations}

This systematic review has several limitations. One limitation is that it is unknown whether follow-up data was complete in all articles which led to an unclear quality assessment. Some articles reported that follow-up data was not complete and this could influence the reliability of the outcome measures. Moreover, different surgeon volume quartiles gave different definitions of high and low volume between studies and therefore a meta-analysis was not possible. Further, the amount of overlap in the US cardiac surgery databases included in this study is unknown but it seems that a proportion of patients are duplicate. This implies that less patients are included than it seems, which makes the data less significant. It is also unknown how many MV surgery patients Ch'ng et al. included and this also influences the reliability of the outcomes (11).

\section{Implications}

Several potential implications can be drawn from this review. First, MV surgery should preferentially be performed by surgeons with high annual surgery volumes. Therefore, it might be necessary to centralize MV surgery in high-volume hospitals or reference centers. Since volume seems an important factor for better outcomes, 
specific training in $\mathrm{MV}$ repair for cardiac surgeons is important to develop and maintain their surgical skills. For example, this can be done through simulation-based training for cardiac surgery (32). Training was shown to be an essential prerequisite for high quality mitral repair (8). Furthermore, experience is crucial since surgical qualities such as clinical judgment and technical expertise might account for a large part in these volume-outcome relationships (23).

\section{Conclusions}

In conclusion, higher surgeon volume is significantly associated with improved outcomes such as repair rate and mortality. Recommendations for further research would be to systematically review the influence of hospital volume on this association between surgeon volume and improved outcomes.

\section{Acknowledgments}

Funding: None.

\section{Footnote}

Reporting Checklist: The authors have completed the PRISMA reporting checklist. Available at https://dx.doi. org/10.21037/jtd-21-578

Conflicts of Interest: All authors have completed the ICMJE uniform disclosure form (available at https://dx.doi. org/10.21037/jtd-21-578). The authors have no conflicts of interest to declare.

Ethical Statement: The authors are accountable for all aspects of the work in ensuring that questions related to the accuracy or integrity of any part of the work are appropriately investigated and resolved.

Open Access Statement: This is an Open Access article distributed in accordance with the Creative Commons Attribution-NonCommercial-NoDerivs 4.0 International License (CC BY-NC-ND 4.0), which permits the noncommercial replication and distribution of the article with the strict proviso that no changes or edits are made and the original work is properly cited (including links to both the formal publication through the relevant DOI and the license). See: https://creativecommons.org/licenses/by-nc-nd/4.0/.

\section{References}

1. Nkomo VT, Gardin JM, Skelton TN, et al. Burden of valvular heart diseases: a population-based study. Lancet 2006;368:1005-11.

2. De Bonis M, Alfieri O, Dalrymple-Hay M, et al. Mitral valve repair in degenerative mitral regurgitation: state of the art. Prog Cardiovasc Dis 2017;60:386-93.

3. Lazam S, Vanoverschelde JL, Tribouilloy C, et al. Twentyyear outcome after mitral repair versus replacement for severe degenerative mitral regurgitation: analysis of a large, prospective, multicenter, international registry. Circulation 2017;135:410-22.

4. Suri RM, Schaff HV, Dearani JA, et al. Survival advantage and improved durability of mitral repair for leaflet prolapse subsets in the current era. Ann Thorac Surg 2006;82:819-26.

5. Acker MA, Parides MK, Perrault LP, et al. Mitral-valve repair versus replacement for severe ischemic mitral regurgitation. N Engl J Med 2014;370:23-32.

6. Anyanwu AC, Bridgewater B, Adams DH. The lottery of mitral valve repair surgery. Heart 2010;96:1964-7.

7. Mack M, Taggart D. Coronary revascularization should be a subspecialty focus in cardiac surgery. J Thorac Cardiovasc Surg 2019;157:945-7.

8. Bridgewater B, Hooper T, Munsch C, et al. Mitral repair best practice: proposed standards. Heart 2006;92:939-44.

9. Khera R, Pandey A, Koshy T, et al. Role of hospital volumes in identifying low-performing and highperforming aortic and mitral valve surgical centers in the United States. JAMA Cardiol 2017;2:1322-31.

10. Birkmeyer JD, Stukel TA, Siewers AE, et al. Surgeon volume and operative mortality in the United States. N Engl J Med 2003;349:2117-27.

11. Ch'ng SL, Cochrane AD, Wolfe R, et al. Procedurespecific cardiac surgeon volume associated with patient outcome following valve surgery, but not isolated CABG surgery. Heart Lung Circ 2015;24:583-9.

12. McCarthy PM. When is your surgeon good enough? When do you need a "referent surgeon"? Curr Cardiol Rep 2009;11:107-13.

13. Maruthappu M, Gilbert BJ, El-Harasis MA, et al. The influence of volume and experience on individual surgical performance: a systematic review. Ann Surg 2015;261:642-7.

14. Chowdhury MM, Dagash H, Pierro A. A systematic review of the impact of volume of surgery and specialization on patient outcome. Br J Surg 2007;94:145-61.

15. Kidher E, Sepehripour A, Punjabi P, et al. Do bigger 
hospitals or busier surgeons do better adult aortic or mitral valve operations? Interact Cardiovasc Thorac Surg 2010;10:605-10.

16. Liberati A, Altman DG, Tetzlaff J, et al. The PRISMA statement for reporting systematic reviews and metaanalyses of studies that evaluate healthcare interventions: explanation and elaboration. BMJ 2009;339:b2700.

17. Higgins JPT, Green S. editors. Cochrane Handbook for Systematic Reviews of Interventions. Version 5.1.0. The Cochrane Collaboration. 2011. Available online: http:// handbook-5-1.cochrane.org/

18. Bolling SF. Can we predict mitral valve repair rates by individual surgeons' mitral volume? Tex Heart Inst J 2011;38:703-4.

19. Bolling SF, Li S, O'Brien SM, et al. Predictors of mitral valve repair: clinical and surgeon factors. Ann Thorac Surg 2010;90:1904-11; discussion 1912.

20. Badhwar V, Vemulapalli S, Mack MA, et al. Volumeoutcome association of mitral valve surgery in the United States. JAMA Cardiol 2020;5:1092-101.

21. Chikwe J, Toyoda N, Anyanwu AC, et al. Relation of mitral valve surgery volume to repair rate, durability, and survival. J Am Coll Cardiol 2017. [Epub ahead of print]. doi: 10.1016/j.jacc.2017.02.026.

22. Hannan EL, Samadashvili Z, Smith CR, et al. Mitral valve repair versus replacement for patients with preserved left ventricular function without heart failure symptoms. J Thorac Cardiovasc Surg 2019;157:1432-9.e2.

23. Kilic A, Shah AS, Conte JV, et al. Operative outcomes in mitral valve surgery: combined effect of surgeon and hospital volume in a population-based analysis. J Thorac Cardiovasc Surg 2013;146:638-46.

24. LaPar DJ, Ailawadi G, Isbell JM, et al. Mitral valve repair rates correlate with surgeon and institutional experience.

Cite this article as: Akmaz B, van Kuijk SMJ, Sardari Nia P. Association between individual surgeon volume and outcome in mitral valve surgery: a systematic review. J Thorac Dis 2021;13(7):4500-4510. doi: 10.21037/jtd-21-578
J Thorac Cardiovasc Surg 2014;148:995-1003; discussion 1003-4.

25. Weiner MM, Hofer I, Lin HM, et al. Relationship among surgical volume, repair quality, and perioperative outcomes for repair of mitral insufficiency in a mitral valve reference center. J Thorac Cardiovasc Surg 2014;148:2021-6.

26. Cho JS, Kim JY, Rhee RY, et al. Contemporary results of open repair of ruptured abdominal aortoiliac aneurysms: effect of surgeon volume on mortality. J Vasc Surg 2008;48:10-7; discussion 17-8.

27. Hannan EL, Popp AJ, Tranmer B, et al. Relationship between provider volume and mortality for carotid endarterectomies in New York state. Stroke 1998;29:2292-7.

28. Dimick JB, Cowan JA Jr, Stanley JC, et al. Surgeon specialty and provider volumes are related to outcome of intact abdominal aortic aneurysm repair in the United States. J Vasc Surg 2003;38:739-44.

29. Bederman SS, Kreder HJ, Weller I, et al. The who, what and when of surgery for the degenerative lumbar spine: a population-based study of surgeon factors, surgical procedures, recent trends and reoperation rates. Can J Surg 2009;52:283-90.

30. Onyeji IC, Sui W, Pagano MJ, et al. Impact of surgeon case volume on reoperation rates after inflatable penile prosthesis surgery. J Urol 2017;197:223-9.

31. Aquina CT, Kelly KN, Probst CP, et al. Surgeon volume plays a significant role in outcomes and cost following open incisional hernia repair. J Gastrointest Surg 2015;19:100-10; discussion 110.

32. Sardari Nia P, Heuts S, Daemen JHT, et al. The EACTS simulation-based training course for endoscopic mitral valve repair: an air-pilot training concept in action. Interact Cardiovasc Thorac Surg 2020;30:691-8. 


\section{Search string}

(((((((“Mitral Valve Insufficiency”[Mesh]) OR “Mitral Valve Prolapse”[Mesh]) OR “Mitral Valve Stenosis”[Mesh]) OR “Mitral Valve Annuloplasty"[Mesh]) OR mitral valve surgery) OR mitral valve repair)) AND ((((high-volume surgeons) OR lowvolume surgeons) OR surgeon volume) OR clinician volume) OR physician volume)) AND ((((“Cardiac Surgical Procedures/ mortality”[Mesh]) OR “Reoperation”[Mesh]) OR repair rate) OR recovery). 